www.jmscr.igmpublication.org Impact Factor 5.244

Index Copernicus Value: 5.88 ISSN (e)-2347-176x ISSN (p) 2455-0450 crossref DOI: http://dx.doi.org/10.18535/jmscr/v4i6.47

Journal Of Medical Science And Clinical Research

\title{
Evaluation of FBS, T3, T4 \& TSH in Patients with Type-2 Diabetes Mellitus
}

\author{
Authors \\ Dr Rabindra Kumar Mohapatra ${ }^{1}$, Dr Tapan Kumar Mohapatra ${ }^{2}$, Dr Preeti Sharma ${ }^{3}$ \\ ${ }^{1,2}$ Professor, Department Of Biochemistry \\ ${ }^{3}$ AssociateProfessor, Department Of Biochemistry \\ ${ }^{1} \mathrm{Hi}-\mathrm{Tech}$ Medical College \& Hospital Rourkela, Odisha, India \\ ${ }^{2} \mathrm{Hi}$-Tech Medical College \& Hospital Bhubaneswar, Odisha, India \\ ${ }^{3}$ Santosh Medical College \& Hospital, Gaziabad, Uttar Pradesh, India
}

\begin{abstract}
s
Diabetes mellitus (DM) is a common endocrine metabolic disorder and is one of the leading causes of death worldwide. Thyroid disorders are also very common in the general population and are second only to diabetes as the most common condition to affect the endocrine system. As a result, it is common for an individual to be affected by both thyroid diseases and diabetes. Our aim was to compare the prevalence of thyroid dysfunction in type 2 diabetic patients and healthy controls. Thyroid disorders and diabetes mellitus have a common auto immuneetiology, diabetic patients having increased TSH could be due to immunological disturbances. Increase in serum TSH levels in diabetes patients compared to healthy controls is suggestive of diabetic patients probably suffering from subclinical hypothyroidism.

Keywords: Type-2 diabetes mellitus, thyroid hormones \& thyroid disorders.
\end{abstract}

\section{Introduction}

In the ancient Sanskrit Literature, diabetes mellitus was described as "honey-urine disease," associated with gross emaciation and wasting. Diabetes is a global endemic with rapidly increasing prevalence in both developing and developed countries. ${ }^{1}$ Diabetes mellitus is a group of metabolic disease characterized by hyperglycemia resulting from defects in insulin secretion, insulin action, or both. The chronic hyperglycemia of diabetes is associated with longterm damage, dysfunction, and failure of various organs, especially the eyes, kidneys, nerves, heart, and blood vessels. Several pathogenic processes areinvolved in the development of diabetes. These range from autoimmune destruction of the $\beta$-cells of the pancreas with consequent insulin deficiency to abnormalities that result in resistance to insulin action. ${ }^{2}$ The vast majority of cases of diabetes fall into two broad etiopathogenetic categories. In one category, type- 1 diabetes, the cause is an absolute deficiency of insulin secretion. Type- 2 diabetes is a second and more common form of diabetes mellitus. The cause is a combination of resistance to insulin action and an inadequate compensatory insulin secretory response.

In Indian population, 61.3 million people had diabetes in 2011, which is expected to reach 101.2 million by 2030 (International Diabetes Federation) now placing India at second position in world diabetic prevalence. Prevalence of diabetes mellitus has been steadily increasing in urban as well as rural areas in India \& it will be one of the major cause of death in India in $21^{\text {st }}$ 
century. Recent data has illustrated the impact of socio-economic transition occurring in rural India. ${ }^{3}$ Studies have revealed that $10 \%-15 \%$ of Type-2 diabetic patients develop auto antibodies to islet cell antigens within the first 5 years of diagnosis. These individuals represent a special subset of type- 2 diabetics as seen by their deficiency of insulin secretion, elevated frequency of certain HLA genes, elevated frequency of thyroid or gastric auto antibodies and high risk for requiring insulin treatment. ${ }^{4}$

The clinical association of autoimmune diseases has shown that among patients in southern India, autoimmune diseases were diagnosed in $1.68 \%$ of persons with diabetes mellitus. ${ }^{5}$ Diabetic patients have a higher prevalence of thyroid disorders compared to the normal population because patients with one organ-specific autoimmune disease are at risk of developing other autoimmune disorders. ${ }^{6}$ In the general population, approximately $6 \%$ of people have some form of thyroid disorder. However, the prevalence of thyroid disorder increases to over $10 \%$ in people with diabetes. There is a higher occurrence of thyroid diseases, particularly hypothyroidism, among people with Type- 2 diabetes. ${ }^{6,7}$

Thyroid diseases are common in the general population, and the prevalence increases with age. Screening for thyroid dysfunction is indicated in certain high risk groups, such as neonates and the elderly. ${ }^{8,9}$

Hypothyroidism is the most common thyroid disorder in the adult population. Hypothyroidism in humans is caused by insufficient production of thyroid hormone by thyroid gland. By contrast, hyperthyroidism is much less common, with a female-to-male ratio of 9:1. Hyperthyroidism is a condition in which thyroid gland produces too much of the hormone thyroxine. Hyperthyroidism can significantly accelerate body's metabolism, causing sudden weight loss, a rapid or irregular heartbeat, sweating, and nervousness or irritability. $^{10,11}$ The presence of thyroid dysfunction may affect diabetes control. Hyperthyroidism is typically associated with worsening glycemic control and increased insulin requirements. There is underlying increased hepatic gluconeogenesis, rapid gastrointestinal glucose absorption, and probably increased insulin resistance. ${ }^{12}$ Therefore, this study has been carried out to find outany thyroid dysfunction in diabetic cases and hence the importance of its estimation in diabetic patients.

\section{Material and method}

The present study was conducted in Hi-Tech Medical College \& Hospital Rourkela, Odisha, India, during the period from November 2014 to July 2015. The study protocol was approved by the Ethics committee of Hi-Tech Medical College \& Hospital Rourkela. Randomly selected, 35 patients with type- 2 diabetic patients with an age ranged from 30 to 70 years along with 25 healthy controls were studied for following parameters.

1. Plasma fasting blood Sugar by GOD-POD method.

2. Serum T3 by ELISA kit method

3. Serum T4 by ELISA kit method

4. Serum TSH by ELISA kit method

All values were expressed as mean $\pm \mathrm{SD}$. We used student t-test and pearson's correlation coefficient to find the statistical significance. A P-value $<0.05$ was to be considered statistically significant.

\section{Results and Discussion}

Among the 35 type- 2 diabetic patients studied, 17 were males and 18 were females with mean age of $55.5 \pm 6.2$ years. Among the 25 healthy controls studied, 14 were males and 11 were females with mean age of $49.8 \pm 6.5$ years. In our study we observed that the TSH level was increased in diabetic patients $(7.459 \pm 4.672)$ as compared to controls $(3.08 \pm 1.56)$ which was statistically significant. However serum T3 andT4 levels were within the normal range and which was statistically significant.

$\mathrm{DM}$ is a complex and multifactorial disease. The metabolic dysregulation associated with diabetes causes secondary pathophysiologic changes in multiple organ systems that impose a heavy burden of morbidity and mortality from macro vascular and micro vascular complications. ${ }^{13}$ 
Table-1: Comparison of T3, T4 \& TSH in between type-2 diabetic patients and healthy controls.

\begin{tabular}{|l|l|l|l|l|}
\hline Parameters & Normal Range & $\begin{array}{l}\text { Controls } \\
\mathrm{N}=25\end{array}$ & $\begin{array}{l}\text { Diabetics } \\
\mathrm{N}=35\end{array}$ & -value \\
\hline FBS mg/dl & $>126$ & $86.57 \pm 8.14$ & $128.17 \pm 37.92$ & 0.02 \\
\hline $\mathrm{T} 3 \mathrm{ng} / \mathrm{ml}$ & $0.52-1.85$ & $1.29 \pm 0.43$ & $1.570 \pm 0.677$ & NS* \\
\hline $\mathrm{T} 4 \mu \mathrm{g} / \mathrm{dl}$ & $4.4-10.8$ & $7.54 \pm 1.94$ & $7.459 \pm 4.672$ & $\mathrm{NS}$ \\
\hline $\mathrm{TSH} \mu \mathrm{IU} / \mathrm{ml}$ & $0.39-6.16$ & $3.08 \pm 1.56$ & $7.459 \pm 4.672$ & 0.02 \\
\hline
\end{tabular}

$(\mathrm{p}<0.05$ - Statistically significant $) ; * N S$ : Statistically not significant

DM and thyroid disorders are the two common endocrinopathies seen in the adult population. Recent studies have shown a very high prevalence of thyroid dysfunction in type 2 DM. Insulin and thyroid hormones are intimately involved in cellular metabolism and thus excess or deficit of either of them may result in the functional derangement of the other. ${ }^{14}$

Subclinical hypothyroidism is defined asan asymptomatic state characterized by normal free thyroxine and triiodothyronine levels and elevated serum concentration softhyrotropin $(>4 \mu \mathrm{U} / \mathrm{ml})$. Diabetic patients have a higher prevalence of thyroid disorders compared with the normal population. ${ }^{15}$

In our study we found statistically significant elevated value of TSH and normal values of T3and T4 which was correlated with previous studies. 13,14,15 ELNobre et al, in 2002 studied association of type 2 diabetes mellitus with thyroid dysfunction and indicated a higher occurrence of thyroid dysfunction among diabetics when compared with the general population. $12.7 \%$ of diabetics had thyroiddysfunction. $^{16}$

C. E.J. U diong, A et al., in 2007 have shown that $46.5 \%$ of diabetics had abnormal thyroid hormone levels. Twenty six per cent of the diabetics had low levels of thyroid hormones while $19.9 \%$. hadraised levels. ${ }^{17}$

Guang-Ran Yang et $\mathrm{al}^{18}$ have shown that $22.4 \%$ of the diagnosed type 2 diabetes patients had subclinical hypothyroidism. Ina study conducted by C.E.J.U diong et al, out of 161 diabetic patients $26.6 \%$ were hypothyroid and $19.9 \%$ were hyperthyroid patients.

\section{Conclusion}

Thus this study shows the prevalence of abnormal thyroidhorm one level among diabetic subjects. The relationship between thyroid disorders and diabetes mellitus is characterized by a complex interdependent interaction. Since thyroid disorders and diabetes mellitus have a common autoimmune etiology, diabetic patients having increased TSH could be due to immunological disturbances. Increase in serum TSH levels in diabetes patients compared to healthy controls is suggestive of diabetic patients probably suffering from subclinical hypothyroidism. Further studies are required to prove the autoimmune basis of development of hypothyroidism in diabetic patients.

\section{Bibliography}

1. Manoj Kumar Yadav, Dr TK Mohapatra, Dr RK Mohapatraet. al. Study on Glycated Hemoglobin\& lipid profile in Type-2 Diabetes Mellitus. International Journal of Science \& Research (IJSR) 2015;4(6): 1917-1919.

2. American diabetes association. Diagnosis and classification of diabetes mellitus. Diabetes care 2010;33: 562-569.

3. A Ramachandran, AK Das, SR Joshi, CS Yajnik, S Shah, KM Prasanna Kumar. Current Status of Diabetes in India and Need for Novel Therapeutic Agents. JAPI2010; 58:7-9.

4. Syed MA, Barinas-Mitchell E, Pietropaolo SL, Zhang YJ, Henderson TS, Kelley DE et al. Is type 2 diabetes a chronic inflammatory/autoimmune disease? Diabetes Nutr Metab.2002; 15(2):68-83. 
5. Sridhar GR, Nagamani G. Clinical association of Autoimmune diseases with Diabetes Mellitus, Ann N Y Acad Sci. 2002;958:390-2.

6. Practica $\mathrm{Wu}$, Thyroid Disease and Diabetes, Clinical diabetes2000;18(1):38-39.

7. MirellaHage, Mira SZ, Sami TA. Thyroid Disorders and Diabetes Mellitus. Journal of Thyroid Research 2011; 2011, Article ID 439463, 7 pages.

8. Tamar Simpser, Robert Rapaport. Update on Some Aspects of Neonatal Thyroid Disease. J Clin Res PediatrEndocrinol. 2010 September; 2(3): 95-99.

9. Rehman SU, Cope DW, Senseney AD, Brzezinski W. Thyroid disorders in elderly atients. South Med J. 2005 May;98 (5):543-9.

10. Mark P. J. Vanderpump. The epidemiology of thyroid disease.Br. Med Bull 2011;99 (1):39-51.

11. Jeri RR, Stephen FW, Hyperthyroidism: Diagnosis and Treatment. Am Fam Physician. 2005 Aug 15;72(4):623-630.

12. Jennal L. Johnson. Diabetes Control in Thyroid Disease. Diabetes Spectrum 2006;19(3):148-153.

13. Pasupathi P, Chandrasekar V, Kumar US. Evaluation of oxidative stress, antioxidant and thyroid hormone status in patients with diabetes mellitus. J Med 2009;10:606.

14. Sathish R Mohan V. Diabetes and thyroid Disease. Int J DiabDev Countries 2003; 23:120-3.

15. Vahab Fatourechi. Subclinical Hypothyroidism: An Update for Primary Care hysicians. MayoClin Proc.2009 January; 84(1): 65-71.

16. Nobre, EL, Jorge Z, Pratas S, Silva C $\&$ CastroJJ. Profile of the thyroid function in a population with type-2 diabetes mellitus ,British Endocrine Societies, 2002.3:298.

17. Udiong CEJ, Udoh AE, Etukudoh ME. Evaluation of Thyroid function in Diabetes mellitus. Indian Journal of Clinical Biochemistry2007;22(2):74-78.

18. Guang-RY,Jin KY, Lin Z,Yan HA, Jing KL.Association between subclinial hypothyroidism and proliferative diabetic retinopathy in type 2 diabetic patients: A case control study. Tohoku J. Exp.2010; 222:303-310.

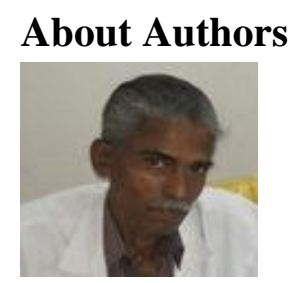

Dr Rabindra Kumar Mohapatra is Professor in Department Of Biochemistry, Hi-Tech Medical College \& Hospital, Rourkela, Odisha.

Email: drrkm.cdr@gmail.com, Mobile: +918895285059

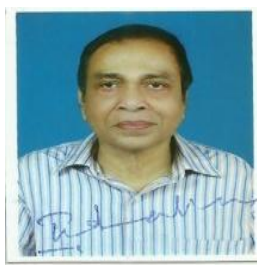

Dr Tapan Kumar Mohapatra is Professor, Department Of Biochemistry, Hi-Tech Medical College \& Hospital, Bhubaneswar, Odisha

Email: tapan.mahapatra7@gmail.com, Mobile: +91-8895798901

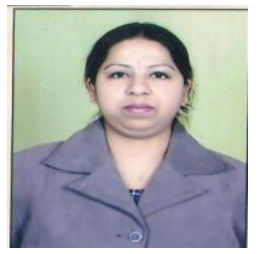

Dr. Preeti Sharma is Associate Professor, Department Of Biochemistry, Santosh Medical College \& Hospital, Gaziabad, Uttar Pradesh, India

Email: prcdri2003@yahoo.co.in, Mobile: +919717248896 